\title{
Time-Resolved Spectral Analysis of Wavelength Conversion by Four-Wave Mixing in Semiconductor Optical Amplifiers
}

\author{
David F. Geraghty, Robert B. Lee, Marc Verdiell, Mehrdad Ziari, Atul Mathur, and Kerry J. Vahala, Member, IEEE,
}

\begin{abstract}
Time-resolved spectral analysis is performed on 10Gb/s signals wavelength converted by four-wave mixing (FWM) in semiconductor optical amplifiers. A pattern-dependent chirp resulting from parasitic gain modulation by the signal is measured and characterized as a function of the converter's pumpto-probe ratio. This chirp is found to be insignificant for pumpto-probe ratios exceeding $9 \mathrm{~dB}$.
\end{abstract}

Index Terms-Communication systems, optical frequency conversion, optical mixers, semiconductor optical amplifiers.

W AVELENGTH conversion has been identified as a key function for the implementation of complex all-optical wavelength-division multiplexed (WDM) communication systems [1]. Many approaches have demonstrated excellent performance [2]-[7]. Of these, only four-wave mixing (FWM) in semiconductor optical amplifiers (SOA's) [2]-[5] offers strict transparency to modulation format and bit rate while providing arbitrary wavelength mapping. Dispersion compensation by optical phase conjugation [8] is also possible using FWM in SOA's [3].

To evaluate the spectral properties of wavelength conversion by FWM in SOA's, we have performed time-resolved spectral analysis (TRSA) [9]. This technique has previously been used to investigate the chirp of modulated lasers [10]. By passing the optical signal through a narrow bandpass filter before detection, the spectral shape of the signal can be determined as a function of time. In this letter, TRSA is performed on $10-\mathrm{Gb} / \mathrm{s}$ signals, both unconverted and converted, generated by directly modulated DFB lasers. The direct modulation will introduce intrinsic chirp into the signal to be analyzed, independent of the wavelength conversion. The experimental setup is shown in Fig. 1. Signal generation and eye diagram analysis are done using a 10-Gb/s bit-error-rate (BER) tester and a microwave transition analyzer. The bandpass filter used is a Hewlett-Packard optical spectrum analyzer with an optical output port. Its bandwidth of $0.8 \AA$ is nonoptimal, but it provides the superior stability required for the measurement. The center wavelength of the filter is stepped by $0.05 \AA$ over

Manuscript received June 23, 1997; revised October 6, 1997. This work was supported by DARPA under Contract DAAL 01-94-K-03430 and by the National Science Foundation under Grant ECS-9412862.

D. F. Geraghty, R. B. Lee, and K. J. Vahala are with the Department of Applied Physics, California Institute of Technology, Pasadena, CA 91125 USA.

M. Verdiell, M. Ziari, and A. Mathur are with SDL, Inc., San Jose, CA 95134 USA.

Publisher Item Identifier S 1041-1135(98)00448-0.

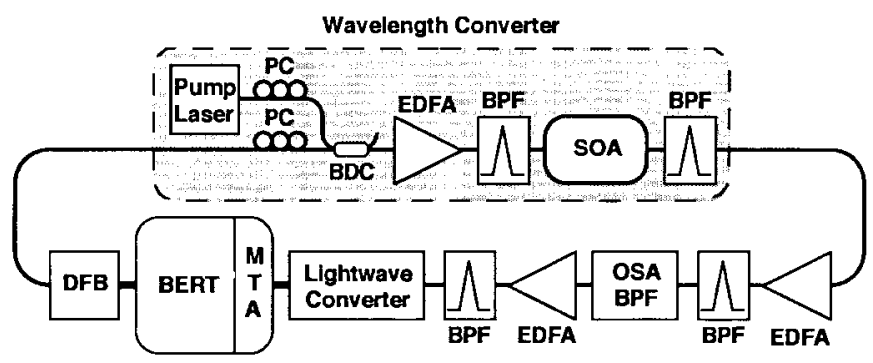

Fig. 1. Experimental setup for TRSA.

the entire spectral width of the signal for each measurement. The filtered signal is then amplified in a high-gain, lownoise erbium-doped fiber amplifier (EDFA) and detected by a Hewlett-Packard lightwave converter consisting of a fast pin detector and an electrical amplifier.

The design of the wavelength converter investigated is also shown in Fig. 1. The wavelength converter's pump source is a tunable, external-cavity semiconductor laser providing about $+3 \mathrm{dBm}$ of in-fiber optical power. Its wavelength is set to provide a 6-nm wavelength downshift. This pump source and the input signal are coupled together in a 50/50 bidirectional coupler after each individually goes through a mechanical polarization controller to align them to the TE axis of the SOA. The combined signals are then amplified in a highpower EDFA and passed through a 10-nm-wide bandpass filter for amplified spontaneous emission prefiltering. The pump and input signal are then coupled into the SOA. The total power input to the SOA is approximately $+13 \mathrm{dBm}$. The SOA is a fiber pigtailed unit from SDL based on a multi quantum-well compressively strained gain medium with 25$\mathrm{dB}$ fiber-to-fiber small-signal gain. Following the SOA, these signals are coupled through a 1-nm-wide bandpass filter to suppress the pump and input signal at the wavelength converter output.

TRSA is performed on the patterns (1000 0000), (1100 0000), (1111 0000), (1011 1000), (0111 1111), (0011 1111) with a pump-to-probe $(\mathrm{P} / \mathrm{Q})$ ratio of $6 \mathrm{~dB}$ in the converter. The data for (1001 1100) is shown in Fig. 2. The complete, unfiltered pattern is shown in Fig. 2(a). Contour plots of constant power with time and wavelength as the the $x$ and $y$ axis, respectively, are shown for both the unconverted and converted signals in Fig. 2(b) and (c). Clearly evident in the contour plots is the spectral inversion resulting from the optical 


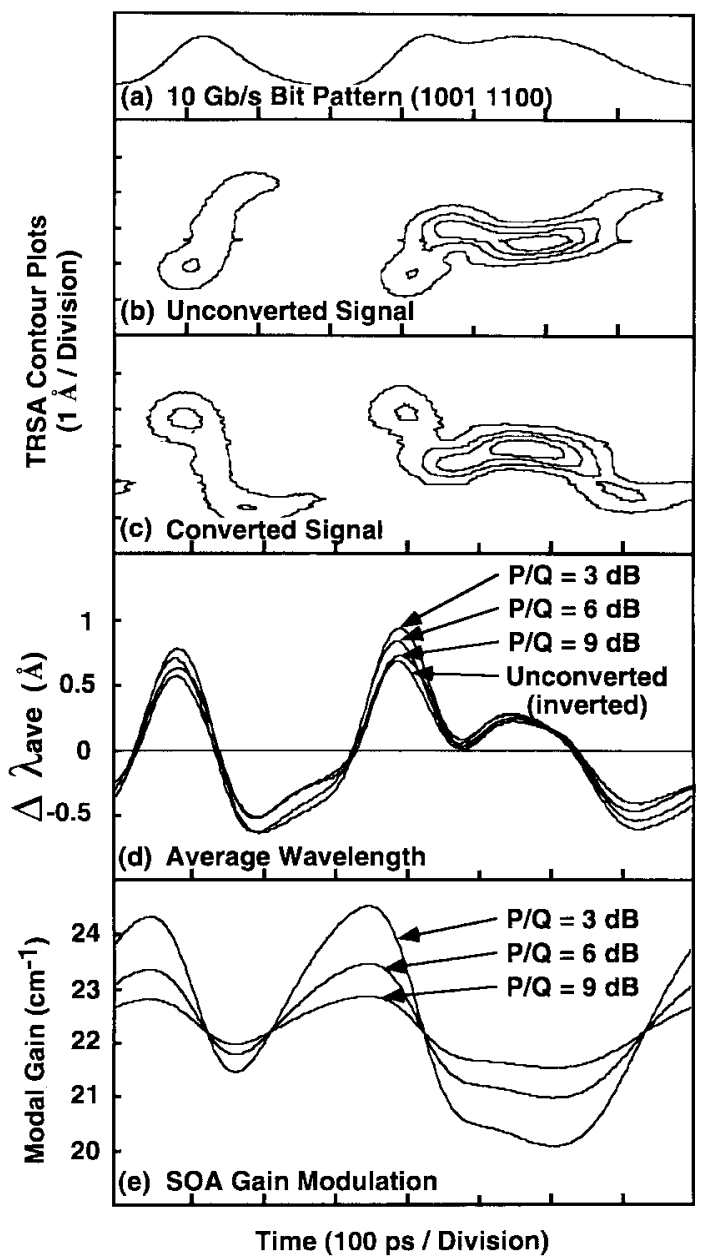

Fig. 2. Time resolved spectral analysis of both the input signal and the converted signal at $10 \mathrm{~Gb} / \mathrm{s}$. (a) The unfiltered $10-\mathrm{Gb} / \mathrm{s}$ bit pattern. Contours plots of constant power with time and frequency as the $x$ and $y$ axis, respectively, for (b) the unconverted and (c) the converted signal. (d) The measured average wavelength of the unconverted signal (inverted) and the converted signal for a P/Q ratios of 3,6, and $9 \mathrm{~dB}$. (e) Predicted SOA gain fluctuations for P/Q ratios of 3,6 , and $9 \mathrm{~dB}$.

phase conjugation. Also apparent is an increase in the spectral shift of the signal after wavelength conversion.

This effect is more easily seen by computing the average wavelength at each point in time. An additional chirp associated with the wavelength conversion can be quantified as the width of the average wavelength increases by $0.27 \AA$, or by $23 \%$, for this pattern. For all the patterns tested with a $\mathrm{P} / \mathrm{Q}$ ratio of $6 \mathrm{~dB}$, the width of the average wavelength increases by 0.047 to $0.47 \AA$, or by 5.5 to $44 \%$. We attribute this additional chirp primarily to parasitic gain saturation and subsequent phase modulation induced by the input signal. The magnitude of this effect will be determined by the pump and probe power levels relative to the SOA saturation power. For our wavelength converter design, this is determined by the $\mathrm{P} / \mathrm{Q}$ ratio since the amplifier is deeply saturated by the pump. The measured average wavelength for a (1001 1100) pattern for P/Q ratios of 3, 6, and $9 \mathrm{~dB}$ is shown in Fig. 2(d), revealing increases of $0.38,0.27$, and $0.054 \AA$, or of $31.5 \%$, $22.8 \%, 4.5 \%$. Also shown is the average wavelength of the unconverted signal (inverted for easy comparison). This chirp seems smaller than that of the raw data in Fig. 2(b) and (c). This results from the spectral-power-weighted averaging done in calculating the average wavelength, as there is low-power signal spanning the intermediate wavelengths throughout the bit pattern.

As noted above, this additional chirp is introduced primarily by parasitic input signal modulation of the SOA gain. Neglecting the transit time of the signal in the SOA and assuming a spatially independent gain, a simple analysis of signal amplification yields the equations [11]

$$
\begin{aligned}
& \frac{d g}{d t}=\frac{g_{0}-g}{\tau_{s}}-\frac{g}{\tau_{s}}\left[\frac{P}{P_{\text {sat }}}\right]^{1.2} \\
& \frac{d \phi}{d t}=-\frac{1}{2} \alpha \frac{d g}{d t} L
\end{aligned}
$$

where $g$ is the saturated modal gain, $g_{0}$ is the unsaturated modal gain, $\tau_{s}$ is the spontaneous lifetime, $P$ is the total power in the SOA, $P_{\text {sat }}$ is the saturation power of the SOA, $\phi$ is the phase of the signal, $\alpha$ is the linewidth enhancement factor, and $L$ is the length of the SOA. The exponent of 1.2 has been added to the gain saturation term to more accurately match the measured saturation dependence, which is not simply linear due to the compressively strained quantum wells [12]. Numerically integrating these equations using parameters determined by gain versus power measurements, the modal gain modulation produced by a (1001 1100) pattern can be calculated. They are shown in Fig. 2(e) for P/Q ratios of 3, 6, and $9 \mathrm{~dB}$. Fluctuations of 4.45, 2.50, and $1.33 \mathrm{~cm}^{-1}$ are predicted. These gain modulations produce phase fluctuations through the linewidth enhancement factor. The phase fluctuations predicted for a linewidth enhancement factor of $\alpha=7$ agree well with the additional chirp measured on the converted signal.

Although not necessarily detrimental for wavelength converison switching between channels of a WDM system, parasitic chirping upon optical phase conjugation in a dispersion compensation system is a serious problem. The above results show that minimizing the parasitic chirp requires operating at larger $\mathrm{P} / \mathrm{Q}$ ratios. This has previously been shown to additionally minimize inter-symbol interference, optimizing the BER performance [13]. This has the unintended effect of reducing the optical signal-to-noise of the converted signal. For example, operating at a large $\mathrm{P} / \mathrm{Q}$ of $9-\mathrm{dB}$ results in an optical signal-to-noise that is over $2 \mathrm{~dB}$ lower than the optimum. Consequently, successful dispersion compensation by SOAbased optical phase conjugation involves careful selection of the P/Q ratio to minimize the parasitic chirping while maximizing the optical signal-to-noise.

\section{ACKNOWLEDGMENT}

The authors acknowledge Dr. Al Benzoni for invaluable discussion and assistance.

\section{REFERENCES}

[1] B. Alexander, "A precompetitive consortium on wide-band all-optical networks," J. Lightwave Technol., vol. 11, pp. 714-732, 1993. 
[2] J. Zhou, N. Park, J. W. Dawson, K. J. Vahala, M. A. Newkirk, and B. I. Miller, "Efficiency of broadband four-wave mixing wavelength conversion using semiconductor traveling-wave amplifiers," IEEE Photon. Technol. Lett., vol. 6, pp. 50-52, 1994.

[3] M. C. Tatham, X. Gu, L.D. Westbrook, G. Sherlock, and D. M. Spirit, "Transmission of $10 \mathrm{~Gb} / \mathrm{s}$ directly modulated DFB signals over $200 \mathrm{~km}$ standard fiber using mid-span spectral inversion," Electron. Lett., vol. 30, pp. 1335-1336, 1994.

[4] R. Ludwig, and G. Raybon, "BER measurements of frequency converted signals using four-wave mixing in a semiconductor laser amplifier at 1 , 2.5, 5, and 10 Gbit/s," Electron. Lett., vol. 30, pp. 338-339, 1994.

[5] D. F. Geraghty, R. B. Lee, K. J. Vahala, M. Verdiell, M. Ziari, and A. Mathur,"Wavelength conversion up to $18 \mathrm{~nm}$ at $10 \mathrm{~Gb} / \mathrm{s}$ by four-wave mixing in a semiconductor optical amplifier," IEEE Photon. Technol. Lett., vol. 9, pp. 452-454, 1997.

[6] J. M. Wiesenfeld, "Gain dynamics and associated nonlinearities in semiconductor optical amplifiers," in Current Trends in Optical Amplifiers and Their Applications T. P. Lee, Ed. New York: World Scientific, 1996.

[7] K. Inoue, "Four-wave mixing in an optical fiber in the zero-dispersion wavelength region," J. Lightwave Technol., vol. 10, pp. 1553-1561, 1992.
[8] A. Yariv, D. Fekete, and D. M. Pepper, "Compensation for channel dispersion by nonlinear optical phase conjugation," Opt. Lett., vol. 4 pp. 52-54, 1979.

[9] D. F. Geraghty, R. B. Lee, K. J. Vahala, M. Verdiell, M. Ziari, and A. Mathur, "Time resolved spectral analysis of phase conjugation by four-wave mixing in semiconductor optical amplifiers," presented at the Conf. Lasers and Electro-Optics, Baltimore, MD, May 18-23, 1997, paper CTuH6.

[10] R. A. Linke,"Modulation induced transient chirping in single frequency lasers," IEEE J. Quantum Electron., vol. QE-21, pp. 593-597, 1985.

[11] G. P. Agrawal and N. A. Olsson, "Self-phase modulation and spectral broadening of optical pulses in semiconductor laser amplifiers," IEEE J. Quantum Electron., vol. QE-25, pp. 2297-2305, 1989.

[12] K. J. Vahala and C. E. Zah, "Effect of doping on the optical gain and the spontaneous noise enhancement factor in quantum well amplifiers and lasers studied by simple analytical expressions," Appl. Phys. Lett. vol. 52, pp. 1945-1947, 1988

[13] M. A. Summerfield and R. S. Tucker, "Optimization of pump and signal powers for wavelength converters based on FWM in semiconductor optical amplifiers," IEEE Photon. Technol. Lett., vol. 8, pp. 1316-1318, 1996. 